\title{
Descripción morfométrica Trichomycteridae (Siluriformes) de las cuencas hidrográficas de la cordillera Chongón-Colonche, provincia de Santa Elena, Ecuador
}

\author{
Morphometric description of the Trichomycteridae family of the \\ hydrographic basins of the Chongon - Colonche mountains.
}

\section{Pedro Guillermo Ramos Montoya ${ }^{{ }^{*}}$ \& Antonio Torres Noboa ${ }^{2}$}

\author{
${ }^{1}$ Consultor independiente \\ ${ }^{2}$ Docente investigador de la Facultad de Ciencias Naturales, Universidad de \\ Guayaquil, Av. Raúl Gómez Lince s/n y Av. Juan Tanca Marengo, Guayaquil, Ecuador.
}

Recibido de abril 2020; recibido en forma revisada 26 de mayo 2020, aceptado 1 de junio 2020

Disponible en línea 26 de junio 2020

\begin{abstract}
Resumen
La presencia de diferentes grupos de peces de agua dulce son el resultado de eventos de especiación en la costa ecuatoriana, esto debido a los factores geomorfológicos y variaciones climáticas. La familia Trichomycteridae es un representante de este proceso evolutivo, sin embargo, existen escasos estudios. Este trabajo describió e identifico a los representantes de dicha familia en dos comunas en la cordillera Chongón - Colonche. Las especies identificadas fueron; 30 individuos para Trichomycterus taenia para Dos Mangas y 34 individuos como Trichomycterus cf. taenia para Loma Alta, mediante regresiones lineales se determinó que las relaciones entre las variables analizadas evidenciaron una tendencia lineal con un coeficiente de correlación superior $a \mathrm{R}=0.89$. Los valores de las pendientes (b) demostraron un crecimiento alométrico negativo $(b<1)$ en todas las regresiones. Se realizó un análisis de variación morfométrica donde se observó una disminución en longitud y ancho de la cabeza en especímenes de la comuna Loma Alta, lo que se presume es que los especímenes recolectados en Loma Alta podrían ser un morfotipo de Trichomycterus taenia.
\end{abstract}

Palabras claves: Trichomycteridae, relaciones morfométricas, crecimiento alométrico, morfométria geométrica.

\begin{abstract}
The presence of different groups of freshwater fish are the result of speciation events in the Ecuadorian coast, this due to geomorphological factors and climatic variations. The Trichomycteridae family is a representative of this evolutionary process, however, there are limited studies. In this work, the representatives of this family in two communes in the Chongón - Colonche mountain were described and identified. The identified species were; 30 individuals for Trichomycterus taenia for Dos Mangas and 34 individuals such as Trichomycterus cf. Taenia for Loma Alta, through linear regressions, it was determined that the relationships between the analyzed variables evidenced a linear trend with a correlation coefficient greater than $R=0.89$. Slope values (b) demonstrated negative allometric growth $(b<1)$ in all the regressions. A morphometric variation analysis was performed where a decrease in head length and width was observed in specimens from the Loma Alta commune, so it is presumed that the specimens collected in Loma Alta may be a possible morphotype of Trichomycterus taenia.
\end{abstract}

Keywords: Trichomycteridae, morphometric relationships, allometric growth, geometric morphometry.

\section{Introducción}

Los ecosistemas estuarinos y dulceacuícolas de Ecuador se han visto modificados por varios eventos geológicos de distinta magnitud. Ejemplo de ello tenemos el levantamiento de la cordillera de los Andes hace aproximadamente 10 millones de años (Duellman, 1979) y el cierre del "Portal de Guayaquil"

* Correspondencia del autor:

E-mail: antonio.torresn@ug.edu.ec hace 8 millones de años (Katzer, 1903). Estos eventos originaron nuevas vertientes y determinaron la dirección de los drenajes del occidente y oriente del país, además, generaron cambios orográficos e hidrográficos que modificaron los parámetros físicos químicos de las cuencas y subcuencas (Barriga, 2012; Antonelli et al., 2009). Estos sucesos han ocasionado especiación en diferentes grupos taxonómicos, entre 
ellos, en los peces dulceacuícolas (Cadena et al., 2012), restringiéndolos a una cuenca determinada. La presencia de diferentes grupos de peces de agua dulce en la costa ecuatoriana son el resultado de eventos de especiación alopátrica (Cadena et al., 2012), esta ocurre cuando una población se subdivide debido a la presencia de una barrera geográfica que promueve el aislamiento espacial de grupos de individuos de una misma especie, que constituían parte de una población original (Mayr, 1963). El proceso de especiación se facilita debido a que los peces son incapaces de cruzar un obstáculo y debido a esto no logran unirse con el resto de la población (Fernández y Schaeffer, 2005).

Las variaciones climáticas y los factores geomorfológicos han influenciado en la evolución de varias especies que ahora son consideradas endémicas en el entorno lótico ecuatoriano, situación que presenta la familia Trichomycteridae la que requiere un análisis particular para aclarar el proceso de especiación (Barriga, 2012). Esta familia está conformada por peces siluriformes de tamaños pequeños, entre sus características generales encontramos que poseen cuerpo sin escamas, una espina dorsal, carecen de aleta adiposa, entre otros. Son conocidos como bagres del Neotrópico, su distribución va desde Centroamérica, Costa Rica hasta Chile en Sudamérica (Arratia, 1983; Pinna y Wosiacki, 2003). En Ecuador están presentes 32 especies de la familia objeto de estudio, tanto al Oriente como al Occidente (Barriga, 2012). En la última década se ha descrito un 25\% de las 298 especies válidas (Frickle et al., 2021).

La escasez de estudios en esta familia probablemente esté relacionada a la falta de interés comercial, debido a que no son utilizados para la alimentación por ser de tamaños pequeños y de contextura delgada, alcanzando un máximo de $70 \mathrm{~mm}$ en adultos (Jiménez-Prado et al., 2015). Por otra parte, la complejidad con el acceso a los ecosistemas habitados por estos individuos es otro factor implicado, debido que se encuentran en ríos alejados de ciudades y muchas veces rodeados por bosques muy densos y de grandes dimensiones. En cuanto a la captura de especímenes es otro limitante debido a que los ríos poseen un grado de inclinación elevado, aumentando la velocidad de la corriente y dificultando los muestreos.

La familia previamente mencionada es un buen representante para evaluar eventos de especiación. Sin embargo, existe escasos estudios que analicen las variaciones morfológicas o genéticas, es importante determinar si se trata de especies diferentes o morfotipos que poseen una distribución más amplia, que podrían existir producto del cambio latitudinal, parámetros físicos químicos y diferentes áreas de drenaje y vertientes. En consecuencia, ante la falta de interés científico es probable que se pierdan especies que aún no han sido descritas.
La relevancia de esta investigación recae en la posibilidad de actualizar rangos de distribución de la familia en estudio o caso contrario el registro de especies endémicas, la información que se generará podrá ser empleada como base para fines científicos en estudios posteriores direccionados a conocer su diversidad, estructuras morfológicas entre otros aspectos que nos faciliten su diferenciación entre individuos de la misma familia o entre familias de otras especies, debido a que las referencias disponibles en el país son insuficientes. En la misma línea, nos dará indicios sobre aquellos factores que significan una amenaza para la diversidad de este grupo y el estado actual de conservación de las especies que conforman la familia en mención. Ecuador posee 32 especies distribuidas tanto al Oriente como al Occidente, no obstante, no se ha encontrado trabajos de línea base enfocados en distribución, proporción sexual, ecología trófica y se desconoce sobre sus aspectos reproductivos (Burgess, 1989), que involucren a la familia Trichomycteridae. Este trabajo se enfocará en describir la morfometría a los representantes de la familia Trichomycteridae de las cuencas hidrográficas de la cordillera Chongón-Colonche.

\section{Antecedentes}

Para la familia Trichomycteridae se encuentran estudios reportados para la zona sur del continente americano, la mayor cantidad de información procede de países como Argentina, Colombia y Chile. No obstante, en Bolivia, Venezuela y Ecuador existe información publicada en menor porcentaje.

Maldonado-Ocampo (2005) describieron a los representantes de la familia Trichomycteridae en su guía de campo de Peces de los Andes colombianos, información que ha sido utilizada como línea base para la descripción de las especies registradas en Ecuador.

Pardo et al. (2005) expresaron que las características ambientales asociadas a las vertientes podrían estar modificando el morfotipo de los peces del género Trichomycterus que habitan en estas cuencas, diferenciándolas entre ellas. En la provincia de Titicaca, los ríos se caracterizan por ser cortos y de bajo caudal, todo lo contrario, ocurre en la provincia Chilena donde se presentan caudales de mayor tamaño y extensión.

Ardila-Rodríguez (2007) registró una nueva especie de Trichomycterus en la cuenca alta del río Lebrija, Trichomycterus ruitoquensis la cual se diferencia de las otras especies de la zona por poseer tres hileras de manchas en su cuerpo. Además, Ardila en el año 2011, describió a Trichomycterus maldonadoi, como nuevo registro para Colombia; que se caracteriza por su pequeño tamaño $(32,1 \mathrm{~mm})$.

Barriga (2012) expresó que en el piedemonte ecuatoriano se evidencia un curso de especiación 
simpátrica en donde se originan nuevas especies en la misma localidad, a pesar de no presentar una barrera geográfica, por lo tanto, recomendó realizar un análisis particular, para aclarar el proceso de especiación de la familia Trichomycteridae.

A manera de complemento, Ecuador posee 32 especies distribuidas tanto al Oriente como al Occidente, no obstante, no se ha encontrado trabajos de línea base enfocados en distribución, proporción sexual, ecología trófica y se desconoce sobre sus aspectos reproductivos (Burgess, 1989), que involucren a la familia Trichomycteridae.

Jiménez-Prado et al. (2015) refirió a Ituglanis laticeps, Trichomycterus taenia, Trichomycterus taczanowskii, Trichomycterus banneaui. cómo los 4 representantes de la familia Trichomycteridae para la zona occidental de Ecuador,

\section{Materiales y Métodos}

\section{Área de estudio}

La zona de estudio son las comunas Loma Alta y Dos Mangas ubicadas es la provincia de Santa Elena, en la costa de Ecuador. La comuna Loma Alta se localiza en la cordillera Chongón-Colonche, al sur del Parque Nacional Machalilla, aproximadamente a $10 \mathrm{~km}$ de la comuna Valdivia. En la comuna existe la Reserva Ecológica Comunal de Loma Alta, esta fue establecida por la misma comunidad para proteger las fuentes de agua, evitar invasiones y pérdidas de tierras. Posee un río denominado Río California que presenta caudales con ecosistemas lénticos y de poca profundidad, áreas boscosas con abundante neblina o garúa en la zona montañosa y vegetación seca en las partes más bajas de la cordillera.

La comuna Dos Mangas está asentada en las laderas montañosas de la cordillera Chongón-Colonche posee gran cantidad de recursos naturales y se encuentra localizada a $7 \mathrm{Km}$ del noreste de Manglaralto. El nombre se le da por la unión de los ríos Grande y Colín, que nacen de las montañas y se cruzan a lo largo de los senderos del bosque (Tandazo y Portocarrero, 2014), éstos poseen características similares, caudales con ecosistemas lénticos de poca profundidad y fondos generalmente pedregosos. La frondosa vegetación corresponde al ecosistema de bosque seco tropical, en la parte alta se encuentran los bosques de garúa que se caracterizan por tener precipitaciones entre 1000 y $1500 \mathrm{~mm}$ por año y con una temperatura promedio inferior de $23^{\circ} \mathrm{C}$.

Las zonas de muestreos (Fig. 1) fueron georreferenciadas con ayuda de un Global Position System (GPS) marca Garmin, a la par se realizó un análisis in situ de los parámetros fisicoquímicos del agua, para la determinación del potencial de hidrógeno $(\mathrm{pH})$ se empleó un $\mathrm{pH}$-metro EcoTestr $\mathrm{pH}$ 2 , la medición de la salinidad se la realizó por medio de un refractómetro portable ATC, por último, para la lectura de la temperatura se utilizó un termómetro digital.

\section{Colecta y preservación de muestras.}

En los sitios de estudio se capturó la ictiofauna por medio de uso de artes de pesca como chinchorro o challo en función de las características hidrográficas de la zona de muestreo. Posterior a eso, se identificaron los organismos pertenecientes a la familia Trichomycteridae que fueron colocados en bolsas plásticas con agua del medio donde fueron recolectados, luego se procedió a colocar oxígeno y sellar la bolsa para su traslado a la ciudad de Guayaquil donde se encuentra el Laboratorio de Ictiología e Investigación de Recursos Bioacuáticos (LIIRBA) de la Facultad de Ciencias Naturales donde se fijaron en formol al $10 \%$ y se preservaron en alcohol al 70\% (Ortega-Lara et al., 1999).

\section{Identificación de especímenes.}

Para la identificación de los especímenes colectados se utilizó claves para la determinación de las especies pertenecientes a la Familia Trichomycteridae en la vertiente occidental del Ecuador (modificado de Eigenmann, 1918). Para complementar la identificación se realizó observación directa de las características descriptivas de los especímenes colectados, comparándolos con la información de la familia Trichomycteridae encontrada en la guía de campo de peces de los Andes de Colombia (MaldonadoOcampo et al., 2005).

\section{Relaciones morfométricas}

Para cada espécimen colectado se utilizó un calibrador para a toma de las siguientes medidas: Longitud total (LT), Longitud estándar (LE), Altura del cuerpo (AlCu), Longitud predorsal (LpreD), Longitud preanal (LPreAn), Longitud prepélvica (LPrePelv), Altura pedúnculo caudal (AlpedCau), Longitud pedúnculo caudal (LpedCau), Longitud base aleta dorsal (LBasAlDor), Longitud base aleta anal (LBasAlAn), Longitud cabeza (LC), Ancho cabeza (AnCa), Altura cabeza (AlCa). Los datos se recopilaron en Excel y en el programa se determinó las relaciones morfométricas de las variables citadas con la LT. Para el análisis de crecimiento se utilizó una fórmula de regresión lineal simple:

$$
y=a+b x
$$

En la que el parámetro "a" es el intercepto de "y"; "b" es la pendiente del modelo. La interpretación de los datos resultantes se llevará de la siguiente manera: Si los valores resultantes de b es 1 esto significa que su cambio es isométrico, es decir, el crecimiento es relativo entre las dos variables analizadas. Si el valor es superior a 1 se obtendrá un crecimiento alométrico positivo, si el valor de b es inferior a 1 , el crecimiento es alométrico negativo. Los datos fueron recopilados en una hoja de cálculo en Excel y se hizo un ajuste de mínimo de cuadrados a los datos mediante la herramienta Solver. Se determinaron coeficientes de correlación lineal $(R)$ donde los valores varían de -1 donde la correlación es inversa, 0 donde no hay 
Rev. Cient. Cien. Nat. Ambien. 14(1):119-126 Junio 2020
Ramos \& Torres • Descripción morfométrica Trichomycteridae (Siluriformes) de las cuencas hidrográficas de la cordillera Chongón-Colonche, provincia de Santa Elena, Ecuador

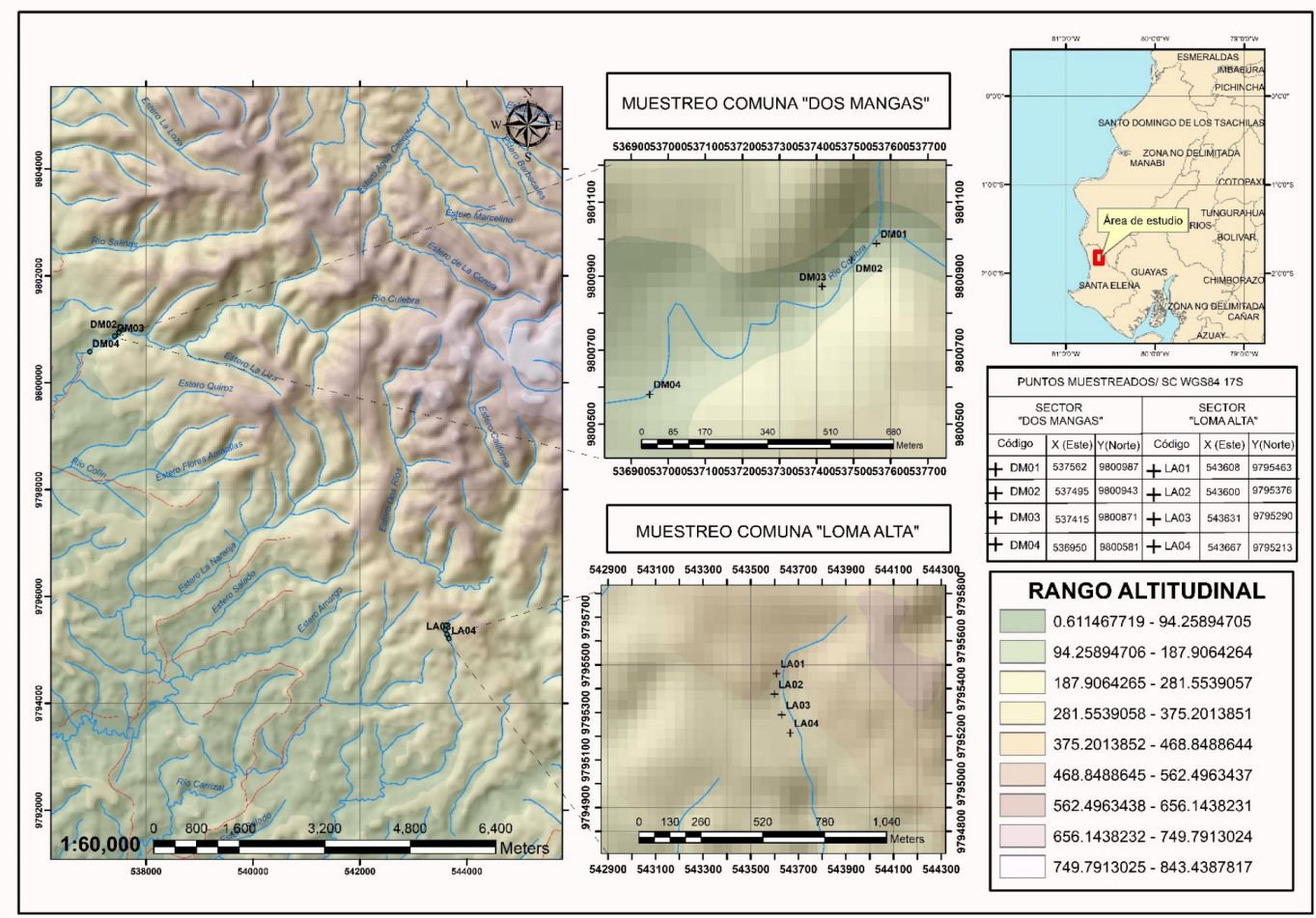

Figura 1. Zonas de muestreo de especímenes de Trichomycteridae en la provincia de Santa Elena.

correlación y 1 donde la correlación es directa. Todos los análisis se realizaron en la mencionada hoja de cálculo.

\section{Morfometría geométrica}

Para determinar diferencias morfológicas se fotografiaron las muestras mediante una cámara profesional Nikon D5300 Profesional, se marcaron 7 puntos o hitos (Fig. 2) de acuerdo con lo establecido para las especies del género Trichomycterus (Pardo et al., 2005). Posteriormente, se utilizó los programas Tps Util y Tps Dig2 para la transformación de las imágenes en coordenadas X, Y. (Aguirre y Jiménez-Prado, 2018). Adicionalmente, a las imágenes transformadas en coordenadas se les realizó el análisis de superposición de Procrustes (PS) que utiliza los métodos de mínimos cuadrados para trasladar, rotar y escalar los hitos a una talla promedio, que muestra la forma pura de la especie, con la ayuda del programa Tps Relw (Rohlf, 2015; Rohlf y Slice, 1990). Luego se llevó a cabo un análisis de componentes principales (PCA) que utiliza los datos del PS para realizar deformaciones parciales, examinando los cambios en la forma de los organismos de cada población a un organismo promedio (Zelditch et al., 2004). Además, se aplicó un análisis de función discriminante, que correlaciona las poblaciones, permitiendo observar la similitud entre los grupos (Gonzáles-Acosta, 2005). Finalmente, se efectuó un agrupamiento por distancias euclidianas utilizando el algoritmo UPGMA con los datos de los componentes principales. Para los análisis mencionados se usará el programa MorphoJ (Aguirre y Jiménez-Prado, 2018).

\section{Resultados}

\section{Identificación}

Un total de 64 individuos fueron recolectados en las zonas de muestreos: 30 especímenes identificados como Trichomycterus taenia (Fig. 3, Tabla 1) para la Comuna Dos Mangas, donde la temperatura fue $24^{\circ} \mathrm{C}$, $\mathrm{pH} 7.5$ y salinidad $0 \%$; y 34 especímenes identificados como Trichomycterus of. taenia (Fig. 4, Tabla 1) para la Comuna Loma Alta, donde los parámetros fueron:

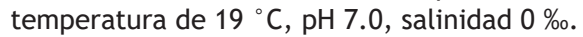

\section{Descripción - Trichomycterus taenia}

A esta especie se la denomina comúnmente como bagrecito (Barriga, 1991) o madre bagre (Laaz y Torres-Noboa, 2014). Presenta su cuerpo alargado y cilíndrico, boca subterminal, ojos en la mitad de la cabeza, máxima longitud total del cuerpo $64 \mathrm{~mm}$,

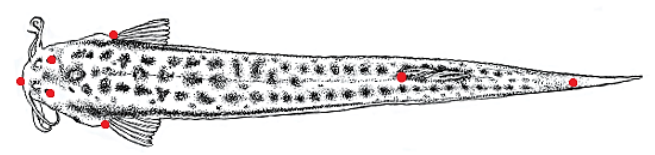

Figura 2. Hitos en espécimen estudiado de la familia Trichomycteridae. 
Tabla 1. Datos morfométricos del holotipo y paratipos de Trichomycterus taenia (Dos Mangas) y T. cf. taenia (Loma Alta).

\begin{tabular}{|c|c|c|c|c|c|c|c|c|c|}
\hline \multirow[b]{2}{*}{ Variables } & \multirow{2}{*}{$\begin{array}{c}\begin{array}{c}\text { Trichomycterus } \\
\text { taenia } \\
\text { (holotipo) }\end{array} \\
\text { Media }\end{array}$} & \multicolumn{4}{|c|}{$\begin{array}{l}\text { Trichomycterus taenia } \\
\text { (Dos Mangas) }\end{array}$} & \multicolumn{4}{|c|}{$\begin{array}{l}\text { Trichomycterus cf. taenia } \\
\text { (Loma Alta) }\end{array}$} \\
\hline & & Min & $\operatorname{Max}$ & Media & DS & Min & $\operatorname{Max}$ & Media & DS \\
\hline LT & 4,76 & 3,30 & 6,60 & 4,76 & 1,07 & 3,60 & 5,20 & 4,26 & 0,49 \\
\hline LE & 4,21 & 2,80 & 5,90 & 4,21 & 1,02 & 3,10 & 4,60 & 3,79 & 0,45 \\
\hline $\mathrm{AlCu}$ & 0,62 & 0,40 & 0,80 & 0,62 & 0,14 & 0,40 & 0,70 & 0,51 & 0,09 \\
\hline Lped & 2,91 & 1,90 & 4,10 & 2,91 & 0,66 & 2,00 & 3,40 & 2,61 & 0,37 \\
\hline LPreAn & 3,04 & 2,00 & 4,00 & 3,04 & 0,62 & 2,40 & 3,50 & 2,84 & 0,35 \\
\hline LPrePelv & 2,48 & 1,60 & 3,40 & 2,48 & 0,51 & 1,80 & 2,90 & 2,29 & 0,30 \\
\hline AlPedCau & 0,49 & 0,40 & 0,70 & 0,49 & 0,11 & 0,30 & 0,60 & 0,42 & 0,08 \\
\hline LPedCau & 0,60 & 0,40 & 0,80 & 0,60 & 0,12 & 0,40 & 0,80 & 0,53 & 0,09 \\
\hline LBasAlDor & 0,45 & 0,20 & 0,40 & 0,45 & 0,08 & 0,20 & 0,30 & 0,24 & 0,05 \\
\hline LBasAlAn & 0,26 & 0,20 & 0,30 & 0,26 & 0,05 & 0,20 & 0,30 & 0,21 & 0,03 \\
\hline LC & 0,66 & 0,50 & 0,90 & 0,66 & 0,13 & 0,40 & 0,80 & 0,56 & 0,10 \\
\hline AnCa & 0,66 & 0,40 & 0,90 & 0,66 & 0,16 & 0,50 & 0,80 & 0,61 & 0,09 \\
\hline $\mathrm{AlCa}$ & 0,44 & 0,30 & 0,60 & 0,44 & 0,09 & 0,40 & 0,50 & 0,39 & 0,05 \\
\hline
\end{tabular}

mínima longitud total del cuerpo $27 \mathrm{~mm}$, su coloración está bien definida, con una banda lateral oscura y ancha que va desde el opérculo hasta el inicio de la aleta caudal (García-Melo, 2005), un par de barbillones nasales alcanzando las espinas operculares; dos pares de barbillones maxilares alcanzando las pectorales (Eigenmann, 1918), el último radio de la aleta dorsal se encuentra por encima del último radio de la aleta anal, característica fundamental para identificación de la especie, aletas dorsales con 7-8 radios, ventrales con 7 radios, pectorales con 7 radios y anales con 5 radios, presenta el pedúnculo caudal delgado y aleta caudal truncada o parcialmente truncada con 15-17 radios para Trichomycterus taenia y con $10-13$ radios para Trichomycterus cf. taenia.

\section{Relaciones morfométricas.}

Para las relaciones morfométricas se correlacionó la variable longitud total LT con las variables restantes, obteniendo los valores que se presentan en la Tabla 2 y Fig. 5. Los resultados de las variables analizadas tienen una tendencia lineal con valores de coeficiente de correlación $(\mathrm{R})$ por encima a 0.89 , como se refleja en la fig. 5. La pendiente (b), en las regresiones lineales, mostraron un crecimiento alométrico negativo ( $b>1$ ), en cada una de las variables evaluadas (Tabla 3). Se determinó que Trichomycterus taenia y

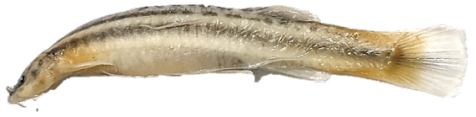

Figura 3. Espécimen de Trichomycterus taenia de Dos Mangas.

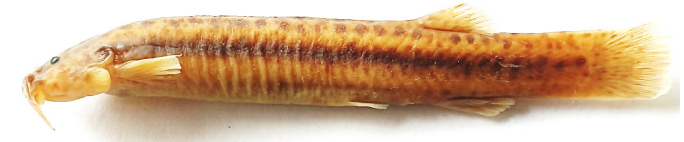

Figura 4. Espécimen de Trichomycterus cf. taenia de Loma Alta.

T. cf. taenia tienen crecimiento de tipo alométrico negativo, esto indica que el crecimiento del pez no va a ser proporcional.

\section{Variación morfométrica}

Al realizar un análisis de función discriminante se evidencia una similitud en varias características morfológicas evaluadas, no obstante, se observa la disminución en la longitud y ancho de la cabeza en la especie de Loma Alta (fig. 6), por lo que se presume que los especímenes recolectados en Loma Alta podrían ser un posible morfotipo de Trichomycterus taenia. 

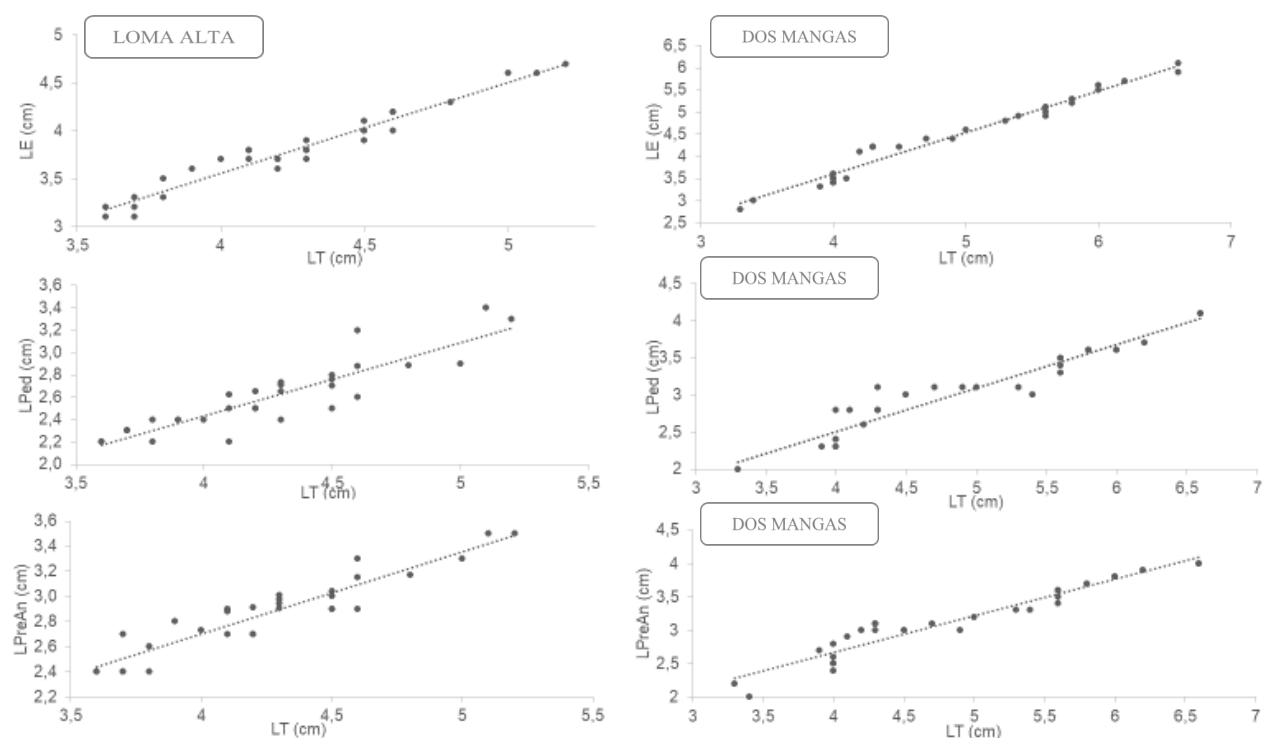

Figura 5. Relaciones morfométricas de las poblaciones de Trichomycterus taenia y T. cf. taenia colectadas en la provincia de Santa Elena.

Tabla 2. La pendiente (b) en ambas poblaciones estudiadas mostró un crecimiento alométrico negativo (b>1).

\begin{tabular}{ccccc}
\hline Variables & $\begin{array}{c}\text { T. cf. taenia } \\
\text { (Loma Alta) }\end{array}$ & Tipo de crecimiento & $\begin{array}{c}\text { T. taenia } \\
\text { (Dos Mangas) }\end{array}$ & Tipo de crecimiento \\
\hline LT-LE & 0,89 & alométrico negativo & 0,90 & alométrico negativo \\
LT-Lped & 0,58 & alométrico negativo & 0,58 & alométrico negativo \\
LT-LPreAn & 0,66 & alométrico negativo & 0,55 & alométrico negativo \\
\hline
\end{tabular}

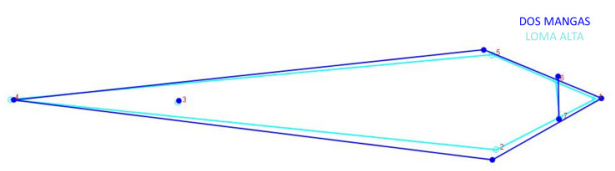

Figura 6. Análisis de función discriminante de las poblaciones de Loma Alta y Dos Mangas.

\section{Discusión}

La información utilizada para la descripción de especies representantes de Trichomycteridae que se encuentran registradas para el Ecuador mayormente se basan en la guía de campo de peces de los Andes colombianos (Maldonado-Ocampo, 2005). La mencionada guía sirvió como un aporte significativo para la elaboración de este trabajo, facilitando la identificación de los especímenes colectados, los cuales de acuerdo a sus características expuestas se ajustaron a la especie Trichomycterus taenia y Trichomycterus cf. taenia.

Durante la realización de este estudio, se presentaron una serie de limitantes que dificultaron el análisis y la interpretación de los resultados obtenidos. Dentro de estas se destacan: el complejo acceso a las zonas de muestreo, variaciones climáticas, factores económicos entre otros.

Se capturaron 64 especímenes de los cuales 30 fueron identificados como Trichomycterus taenia y los 34 restantes como Trichomycterus cf. taenia, descritos para la vertiente occidental del Norte (Eigenman, 1918); en la cuenca baja del Guayas y posiblemente en otras localidades de esta región (Glodek, 1978; Laaz y Torres-Noboa, 2014).

Las relaciones morfométricas en Trichomycterus taenia y Trichomycterus $c f$. taenia mostraron una tendencia lineal con valores aceptables de coeficiente de correlación y determinación, revelaron un crecimiento alométrico negativo en las variables analizadas lo que indica que el crecimiento del pez no va a ser proporcional.

Pardo et al. (2005), expresaron que las características ambientales asociadas a las vertientes podrían estar modificando el morfotipo de los peces que habitan en estas cuencas, diferenciándolas entre ellas, de la misma manera se utilizó la metodología empleada por Pardo et al. en este trabajo, donde se observó una reducción 
en el tamaño de la cabeza de Trichomycterus cf. taenia de Loma Alta. Lo que se prevé es que los especímenes podrían ser un posible morfotipo de T. taenia.

Barriga (2012), expresó que se evidencia un curso de especiación simpátrica en peces de agua dulce y sugirió que la familia Trichomycteridae requiere un análisis particular. Siendo este el caso de Trichomycterus cf. taenia, que fue localizado en las vertientes en la comuna Loma Alta, por lo tanto, se plantea explorar la especie en otras épocas del año y aumentar el tamaño de los datos obtenidos en este estudio.

\section{Conclusión}

La descripción morfométrica de las especies de la familia Trichomycteridae presentes en las cuencas hidrográficas de la cordillera Chongón-Colonche nos permite llegar a las siguientes conclusiones,

Las especies identificadas son Trichomycterus taenia para la comuna Dos Mangas y Trichomycterus cf. taenia para la comuna Loma Alta.

Las relaciones morfométricas de $T$. taenia y $T$. cf. taenia muestran una tendencia lineal, los valores de correlación y determinación fueron aceptables y su crecimiento es alométrico negativo.

Existe variación morfométrica en $T$. cf. taenia de la comuna Loma Alta, este presentó menor tamaño en el ancho y longitud de la cabeza con relación a $T$. taenia de la comuna Dos Mangas

Los especímenes de $T$. cf. taenia podrían ser un morfotipo de T. taenia.

\section{Recomendaciones}

- Realizar análisis genéticos que permitan determinar si Trichomycterus cf. taenia de la comuna Loma Alta es un morfotipo de Trichomycterus taenia.

- Establecer las variables ambientales que influyen en las variaciones morfométricas de las especies de Trichomycteridae.

- Evaluar la biología reproductiva de Trichomycteridae para la cordillera de Chongón-Colonche.

- $\quad$ Analizar el comportamiento de las especies evaluadas en las diferentes la época del año.

- Actualizar las guías de identificación morfológicas para las especies de Trichomycteridae registradas en Ecuador.

\section{Bibliografía}

Aguirre, W., y Jiménez-Prado. 2018. Guía práctica de Morfometría Geométrica. Aplicaciones en la Ictiología. Pontificia Universidad Católica del Ecuador Sede Esmeraldas (PUCESE). Esmeraldas, Ecuador. 104 pp.

Ardila-Rodríguez, C.A. 2007. Trichomycterus ruitoquensis (Siluriformes: Trichomycteridae) una nueva especie de pez de la cuenca alta del Río Lebrija, Departamento de Santander-Colombia. Peces del Departamento de Santander-Colombia, Santander, 3:1-18.

Arratia, G. 1983. Trichomycterus chungaraensis n. sp. and Trichomycterus laucaensis n. sp. (Pisces, Siluriformes, Trichomycteridae) from the high Andean range. Studies on Neotropical Fauna and Environment, 18: 65-87.

Antonelli, A., Nylander, J., Persson, C., y Sanmartín, I. 2009. Tracing the impact of the Andean uplift on Neotropical plant evolution. Proceedings of the National Academy of Sciences of the United States of America, 106: 9749-54.

Barriga, R. 1991. Lista de Vertebrados del Ecuador. Peces de agua dulce. Politécnica 16 Biología 3: 5-88.

Barriga, R. 2012. Lista de peces de agua dulce e intermareales del Ecuador. 1st ed. [ebook] Quito, pp. 96-97. Disponible en: https://www.researchgate.net/.../278036573_Guia_ peces_del_Occidente_de_Ecuador [Acceso 27 Sep. 2018].

Burgess, A. 1989. An atlas of freshwater and marine catfishes. A preliminary survey of the Siluriformes. T.F.H. Publications, Neptune City, New Jersey, U.S.A. 1-784, Pls. 1-285.

Cadena, D., Kozak, H., Gómez, P., Parra, L., McCain, M., Bowie, K., Carnaval, C., Moritz, C., Rahbek, C., Roberts, E., Sanders, J., Schneider, J., VanDerWal, J., Zamudio, R., y Graham, H. 2012. Latitude, elevational climatic zonation and speciation in New World vertebrates. Proceedings of The Royal Society B. Biological Sciences 279: 194-201 pp.

Duellman, E. 1979. The herpetofauna of the Andes: patterns of distribution, origin, differentiation and present communities. En: Duellman, W. E. (ed.), The South American Heipetofauna: Its Origin, Evolution, and Dispersal, pp. 371-459. Monograph of the Museum of Natural History, the University of Kansas, Kansas, USA.

Eigenmann, H. 1918. The Pygidiidae, a family of South American catfishes. Memoirs of the Carnegie Museum, 7: 259-398.

Fricke, R., Eschmeyer, W. N., y Van der Laan, R. (Eds.). 2021. Eschmeyer's Catalog of Fishes: genera, species, references http://research.calacademy.org/research/ ichthyology/catalog/Fishcatmain.asp Electronic version accessed 24 Jun 2021.

Fernández, L., y Schaefer, S. 2005. New Trichomycterus (Siluriformes: Trichomycteridae) from an offshore island of Colombia. Copeia, 2005: 68-76.

García-Melo, L.J. 2005. Distribución, diversidad y ecología básica de la familia Trichomycteridae (Ostariophysi: Siluriformes), en la cuenca del río Coello, departamento del Tolima. Trabajo de Grado. Programa de Biología, Facultad de Ciencias, Universidad del Tolima. Ibagué, Colombia. 149 pp.

González-Acosta, A. 2005. Estudio sistemático y biogeográfico del género Eugerres (Perciformes: Gerreidae). Tesis de Doctorado en Ciencias Marinas. CICIMAR-IPN. México. 206 pp.

Glodek, S. 1978. The freshwater fishes of western Ecuador. Master's Thesis, Northern Illinois University. 415 pp.

Jiménez-Prado, P., Aguirre, W., Laaz-Moncayo, E., NavarreteAmaya, R., Nugra-Salazar, F., Rebolledo-Monsalve, R., Zárate-Hugo, E., Torres-Noboa, A., y Valdiviezo-Rivera, J. 2015. Guía de peces para aguas continentales en la vertiente occidental del Ecuador. Pontificia Universidad Católica del Ecuador Sede Esmeraldas (PUCESE); Universidad del Azuay (UDA) y Museo Ecuatoriano de 
Ciencias Naturales (MECN) del Instituto Nacional de Biodiversidad. Esmeraldas, Ecuador. 416 pp.

Katzer, F. 1903. Grundüge des Geologie des unteren Amazonas-Gebietes des States Pará in Brasilien. Leipzig, Verlag von Max Weg, $298 \mathrm{pp}$.

Laaz, E. y Torres-Noboa, A. 2014. Lista de peces continentales de la cuenca del río Guayas. Disponible en: condor. depaul. edu/waguirre/fishwestec/lista_peces_guayasv2.pdf.

Maldonado-Ocampo, A., Ortega-Lara, A., Usma O., Galvis V., Villa-Navarro, G., Vásquez, G., Prada-Pedreros, L., y Ardila, C. 2005. Peces de los Andes de Colombia. Instituto de Investigación de Recursos Biológicos «Alexander von Humboldt». Bogotá, D.C. Colombia. 346 pp.

Mayr, E. 1963. Animal Species and Evolution. Belknap Press. Cambridge, Mass. USA. 797 pp.

Ortega-Lara, A., Murillo, O., Pimienta, C., y Sterling, E. 1999. Los peces del Alto Cauca, Catálogo de especies. Corporación Autónoma Regional del Valle del Cauca-CVC, Santiago de Cali, Colombia, 122 pp.

Pinna, M., y Wosiacki, W. 2003. Familia Trichomycteridae. REREIS, SO Kullander, CJ Ferraris (eds.). Lista de verificación de los peces de agua dulce de América del Sur y Central. Edipucrs, Porto Alegre. 729 pp.

Pardo, R., Scott, S., y Vila, I. 2005. Análisis de formas en especies chilenas del género Trichomycterus (osteichthyes: siluriformes) utilizando morfometría geométrica. Gayana, 69:180-183.

Rohlf, F. 2015. The tps series of software. 1st ed. [ebook] New York: Stony Brook University, pp. 10-11. Disponible en: http://www.italian-journal-of-mammalogy.it/article/ view/11264/pdf [Acceso 18 Abr. 2019].

Rohlf, F.J., y Slice, D. 1990. Extensions of the Procrustes method for the optimal superimposition of landmark. Systematic Zoology, 39: 40-59 pp.

Tandazo, Y., y Portocarrero, M. 2014. Propuesta para la promoción de la Comuna de Dos Mangas, Provincia de Santa Elena como alternativa de desarrollo turístico local. Tesis de licenciatura. Universidad Católica de Santiago de Guayaquil.

Zelditch, M., Swiderski, L., Sheets, D., y Finl, L. 2004. Geometric Morphometrics for Biologists: A Primer. Elsevier Academic Press, New York, USA, 443 pp. 